\title{
Is it a stroke?
}

\author{
Graeme J Hankey Winthrop professor of neurology; consultant neurologist ${ }^{12}$, David J Blacker clinical \\ professor; consultant neurologist ${ }^{123}$
}

'School of Medicine and Pharmacology, University of Western Australia, Harry Perkins Institute of Medical Research, QEII Medical Centre, Nedlands, Perth, Western Australia, Australia 6009; ${ }^{2}$ Department of Neurology, Sir Charles Gairdner Hospital, Nedlands, Perth, Australia; ${ }^{3}$ Western Australian Neuroscience Research Institute, Nedlands, Perth, Australia

Stroke is increasingly common and often fatal or disabling. ${ }^{1}$ The absence of a definitive diagnostic test for stroke and the potential for emergency interventions to restore brain perfusion, ${ }^{2}$ improve survival free of handicap, and minimise early recurrent stroke $^{3}$ mean that doctors need to be able to diagnose acute stroke rapidly and accurately.

\section{What is a stroke?}

Stroke is not consistently defined in clinical practice, clinical research, and public health. Traditionally, stroke has been defined clinically by the abrupt onset of symptoms of focal neurological dysfunction that last more than 24 hours (or lead to earlier death) and are caused by acute vascular injury to part of the brain. ${ }^{4}$ The vascular causes include inadequate blood supply to part of the brain or spinal cord (ischaemic stroke, arterial or venous) and spontaneous haemorrhage into part of the brain (primary intracerebral haemorrhage) or over the surface of the brain (subarachnoid haemorrhage).

Advances in technology have prompted an updated definition of stroke as an acute episode of focal dysfunction of the brain, retina, or spinal cord of any duration in which imaging (computed tomography or magnetic resonance imaging) or autopsy show focal infarction or haemorrhage relevant to the symptoms. ${ }^{5}$ This definition awaits endorsement, particularly for regions without access to magnetic resonance imaging. ${ }^{46}$

\section{How do patients with acute stroke present?}

Stroke presents in a variety of ways.

\section{Typical presentations: focal neurological symptoms of sudden onset}

Typically, stroke presents spontaneously with the sudden or rapid onset of loss of function of a particular part(s) of the body due to loss of function of a particular part of the brain, retina, or spinal cord.

Common focal, anatomically-localising neurologic symptoms include unilateral weakness (corticospinal tract), unilateral sensory loss (spinothalamic tract), monocular blindness (retina or optic nerve), hemianopic visual field loss (optic radiation), double vision (oculomotor pathways), speech disturbance (dominant hemisphere), visual-spatial-perceptual dysfunction (non-dominant hemisphere), clumsiness or ataxia (cerebellum or its connections), and vertigo (vestibulo-cerebellum), which may appear in isolation or combination.

Associated symptoms vary and usually reflect the cause or a consequence of the stroke. For example, headache occurs in about a quarter of patients with acute ischaemic stroke, half of patients with intracerebral haemorrhage, and nearly all patients with subarachnoid haemorrhage, and may reflect the underlying cause of the stroke (such as cervical artery dissection, giant cell arteritis) or a consequence of the stroke (such as cortical ischaemia, intracranial haemorrhage). ${ }^{4}$

\section{Atypical presentations: stroke "chameleons"}

Less commonly, patients have atypical stroke symptoms (stroke "chameleons") that imitate other neurological diseases. This is because the symptoms

- Are not anatomically localising (such as neuropsychiatric, confusion, depressed consciousness) 


\section{The bottom line}

- Suspect the diagnosis of stroke in all patients with abrupt onset of neurological symptoms, particularly in those with risk factors for stroke. Early and accurate diagnosis of stroke enables early interventions targeted to the cause, which may improve survival and functional recovery and minimise early recurrent stroke.

- Some stroke patients will present with atypical stroke symptoms in which the symptom onset is not sudden or the loss of neurological function is not clearly anatomically-localising.

- The FAST (Facial drooping, Arm weakness, Speech difficulties and Test (or Time)) score is a useful screening test in the community, while emergency department doctors may use the FAST or ROSIER scales, and stroke physicians will undertake a more complete and systematic neurovascular assessment.

- Stroke lacks a perfect diagnostic test, and current diagnosis relies on clinical history and examination, supported by brain imaging (such as computed tomography and magnetic resonance imaging, which can be normal).

- Seizures, syncope, and sepsis account for $20-25 \%$ of suspected strokes.

- Are positive (such as abnormal movements, rather than paralysis, due to seizure, alien hand syndrome, or hemiballismus)

- Seem to be peripheral nerve in origin (vestibular syndrome, other cranial nerve palsy, cortical hand syndrome, monoparesis)

- Are isolated (isolated vertigo, binocular blindness, amnesia, anosognosia, dysarthria, dysphagia, stridor, foreign accent, or headache). ${ }^{78}$

Such atypical symptoms are more likely to be due to a stroke if the patient has known cardiovascular disease or risk factors.

\section{Subarachnoid haemorrhage}

This typically presents with a sudden, severe diffuse headache in nearly all patients, ${ }^{4}$ usually without focal neurological symptoms or signs. Neck stiffness is not invariable and may not occur for hours. Other features include vomiting (75\%), depressed consciousness (67\%), focal neurological dysfunction (15\%), intraocular subhyaloid haemorrhages (14\%), epileptic seizures $(7 \%)$, delirium $(1 \%)$, radicular or precordial pain (spinal subarachnoid haemorrhage), severe hypertension, and electrocardiographic changes that may mimic acute myocardial infarction. ${ }^{9}$

A headache may not be present in patients with subarachnoid haemorrhage and depressed consciousness. Also, other symptoms may prevail over headache in patients with subarachnoid haemorrhage who have a confusional state, epileptic seizure, or associated head trauma.

\section{Cerebral vein thrombosis}

Cerebral vein and venous sinus thrombosis typically presents with gradual onset headache followed by focal neurological deficits, epileptic seizures, or impairment of consciousness, in different combinations and degrees of severity. ${ }^{10}$ In up to $15 \%$ of patients however, the onset of the headache is sudden ("thunderclap headache").

\section{How is stroke diagnosed? Early recognition}

The Face Arm and Speech Test (FAST) is a simple, three item recognition tool to aid screening for stroke in the community (box 1). The presence of acute facial paresis, arm drift, or abnormal speech increases the likelihood of stroke (likelihood ratio of $>1$ finding $=5.5$ (95\% confidence interval 3.3 to 9.1$)$ ), while the absence of all three decreases the likelihood (likelihood ratio of 0 findings $=0.39(0.25$ to 0.61$)){ }^{11}$ Paramedics using FAST achieve high levels of detection and diagnostic accuracy of stroke ${ }^{12}$ but may miss posterior circulation strokes ${ }^{13}$ and treatable stroke mimics such as hypoglycaemia.
Emergency department clinicians may opt for the Recognition of Stroke in the Emergency Room (ROSIER) score, which comprises the three FAST items plus visual field defect, leg weakness, loss of consciousness or syncope and seizure activity (see online data supplement). ${ }^{14}$ An initial study among emergency department physicians showed increased diagnostic sensitivity compared with FAST, ${ }^{14}$ although a later study showed similar sensitivities and specificities for both. ${ }^{15}$

\section{Clinical diagnosis}

Stroke is diagnosed clinically on the basis of a description of sudden onset of loss of focal neurological function that is thought to be due to disturbed blood supply to the relevant part of the brain, retina, or spinal cord. If these criteria are met, the likelihood of a stroke is high because stroke is common (that is, high prevalence or pre-test probability), and even higher if the "milieu" is appropriate (such as an elderly patient with a history of prolonged exposure to vascular risk factors).

Symptoms associated with a high agreement between observers for the diagnosis of stroke versus no vascular event are sudden paralysis or weakness, numbness or tingling, change in speech, visual loss, diplopia, and non-orthostatic dizziness (kappa = 0.60 (95\% confidence interval 0.52 to 0.68$)$ ). ${ }^{11}$

An observational study of 350 presentations with suspected stroke found that eight items in the clinical bedside assessment independently predicted the diagnosis of stroke (see table $\downarrow$ ). ${ }^{16-18}$ Many of these predictors have been validated in other studies. ${ }^{19}$

If the patient is seen within 24 hours of symptom onset, and if the neurological symptoms are still present at the time of the assessment and thought to be vascular in origin, the patient should be managed as if he or she has had a stroke, rather than diagnosing a transient ischaemic attack and predicting that the symptoms will resolve within 24 hours.

\section{Investigations}

The diagnosis of stroke is confirmed by computed tomography or magnetic resonance imaging of the brain or cerebrospinal fluid examination for subarachnoid blood. ${ }^{5}$

\section{Non-contrast computed tomography (CT) of the head}

This is the initial diagnostic standard because it is widely and rapidly available and has near perfect sensitivity for acute intracranial haemorrhage. Ischaemic stroke may not be apparent on the initial CT scan, although subtle signs of early ischaemia are usually evident. The sensitivity of CT for diagnosing acute ischaemic stroke is limited if the focal ischaemia is recent (minutes to hours), small, or in the posterior fossa. The sensitivity of CT for diagnosing subarachnoid haemorrhage is limited if it is small or the CT scan is delayed, which allows 


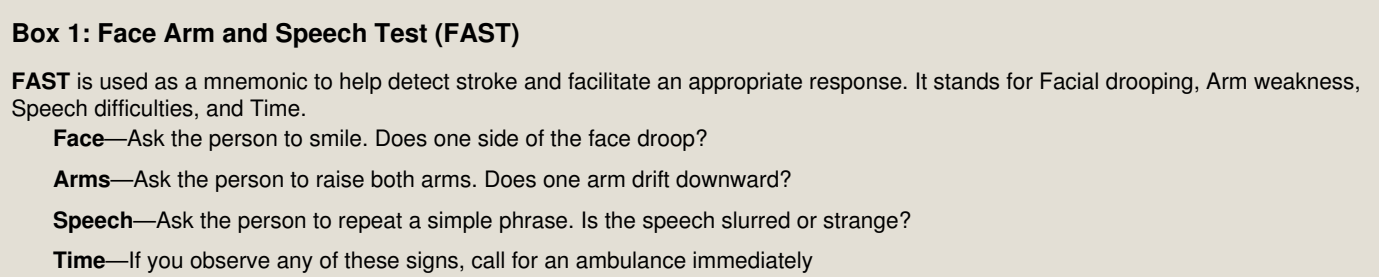

time for the subarachnoid blood to degrade. The sensitivity of CT is $98 \%$ for subarachnoid haemorrhage within 12 hours, $93 \%$ at 24 hours, and declines rapidly after 10 days.

\section{Lumbar puncture and cerebrospinal fluid examination (CSF) examination for subarachnoid blood}

This is required if SAH is being considered and the CT scan is non-diagnostic. ${ }^{9}$ However, the prevalence of xanthochromia in the CSF also declines with time and is detected in only $70 \%$ of cases of subarachnoid haemorrhage after three weeks and $40 \%$ after four weeks.

\section{Magnetic resonance imaging (MRI)}

Diffusion weighted MRI is more sensitive than CT scanning for detecting acute cerebral ischaemia, particularly in patients presenting within 12 hours of symptom onset. ${ }^{20}{ }^{21} \mathrm{~A}$ meta-analysis of eight studies involving 308 participants reported that the sensitivity of diffusion weighted MRI for diagnosing acute ischaemic stroke was 0.99 (95\% confidence interval 0.23 to 1.00 ) and the specificity was 0.92 (0.83 to 0.97 ); the summary estimates for CT were sensitivity 0.39 ( 0.16 to 0.69 ) and specificity 1.00 (0.94 to 1.00$).{ }^{21}$ A caveat of this meta-analysis is that the reported high sensitivity of MRI was obtained in patients with a high pre-test probability of stroke (such as major stroke). ${ }^{21}$ Subsequent studies in patients with minor ischaemic stroke (who tend to have a lower pre-test probability of stroke) have reported the sensitivity of diffusion weighted MRI to be only $76 \%$ ( 71 to $81 \%$ ). ${ }^{22}$ Therefore, a negative diffusion weighted MRI result does not exclude the diagnosis of stroke, particularly in patients with minor ischaemic stroke. ${ }^{22}$ Further, a positive diffusion weighted MRI result does not confirm the diagnosis of stroke ${ }^{23} 24$; it may be due to non-stroke conditions such as seizures, migraine,

hypoglycaemia, tumour, encephalitis, abscess, and multiple sclerosis.

Gradient echo T2-weighted susceptibility MRI is as sensitive as CT for acute haemorrhage and is more sensitive for previous haemorrhage. MRI venography or CT venography is usually required to confirm the diagnosis of cerebral vein and venous sinus thrombosis.

\section{Investigation sequence}

Thus when assessing a patient with suspected acute stroke, an urgent plain CT brain scan often identifies an area of acute focal brain ischaemia and almost always identifies an area of acute focal brain or subarachnoid haemorrhage. If the CT does not show either, then undertake an MRI to identify early infarction (there is no need to specify MRI sequences such as diffusion weighted or gradient echo), or lumbar puncture and CSF examination if subarachnoid haemorrhage is suspected.

To seek the cause of the stroke, imaging of the relevant carotid or vertebral arterial system by carotid ultrasound or CT or MRI angiography, and heart and aortic arch by echocardiography, may be required.

If the patient's symptoms have resolved quickly because of a probable transient ischaemic attack and the CT scan is unremarkable, subsequent tests are still needed to find the underlying cardiovascular cause of the transient ischaemia (such as carotid stenosis or atrial fibrillation) and to treat that urgently to prevent recurrent transient ischaemic attack or fatal or disabling stroke.

\section{What are the diagnostic pitfalls?}

In the community, stroke patients and health professionals do not always recognize the symptoms of stroke. The relationship between the nature of the clinical symptoms and the nature, site, and severity of the underlying brain and vascular pathology are unreliable. Hence, symptoms of a stroke may be attributed to a more benign explanation. Also, focal neurological symptoms of stroke tend to be negative (that is, loss of function) rather than positive. Thus a loss of function may not be noticed until the function is attempted (such as speaking or writing).

Community screening tools such as FAST aim to assist in earlier recognition.

The clinical diagnosis of stroke can be most difficult within the critical first few hours, particularly if the onset of symptoms is uncertain (for example, because of coma, dysphasia, confusion, or no witness), clinical features are atypical ("chameleons") or changing, the patient is too unwell or agitated to obtain a reliable history or interpretable brain imaging, access to imaging is delayed, or the imaging is normal. ${ }^{78}$

Even experienced neurologists show considerable inter-observer variability in the diagnosis of acute stroke, probably because of the inherent limitations of the clinical assessment and brain imaging. ${ }^{11}$ Sometimes the available information does not allow an obvious answer, and the diagnosis is made or excluded on the basis of probability. However, it is important for clinicians to avoid premature closure of the diagnosis during the emergency assessment of a suspected acute stroke. If the initial diagnosis of stroke is not confirmed or not likely, it may be necessary to withdraw it, to avoid inappropriate subsequent investigations, treatments, and consequences (such as implications for driving and insurance policies) and to facilitate "diagnostic vigilance" for other diagnoses ("mimics").

\section{What other conditions may mimic a stroke and lead to "overdiagnosis" of stroke?}

Among patients presenting with a typical stroke syndrome, up to $20-25 \%$ will have a stroke mimic (box 2). ${ }^{85}$ Unfortunately, brain imaging alone does not always distinguish stroke from its mimics, and other investigations may be required to exclude the differential diagnoses shown in box 2 .

Among patients presenting with other neurological syndromes, such as isolated vertigo, stroke may be the underlying cause. A common example is the acute vestibular syndrome (isolated vertigo with secondary ataxia and nausea), which may have a 


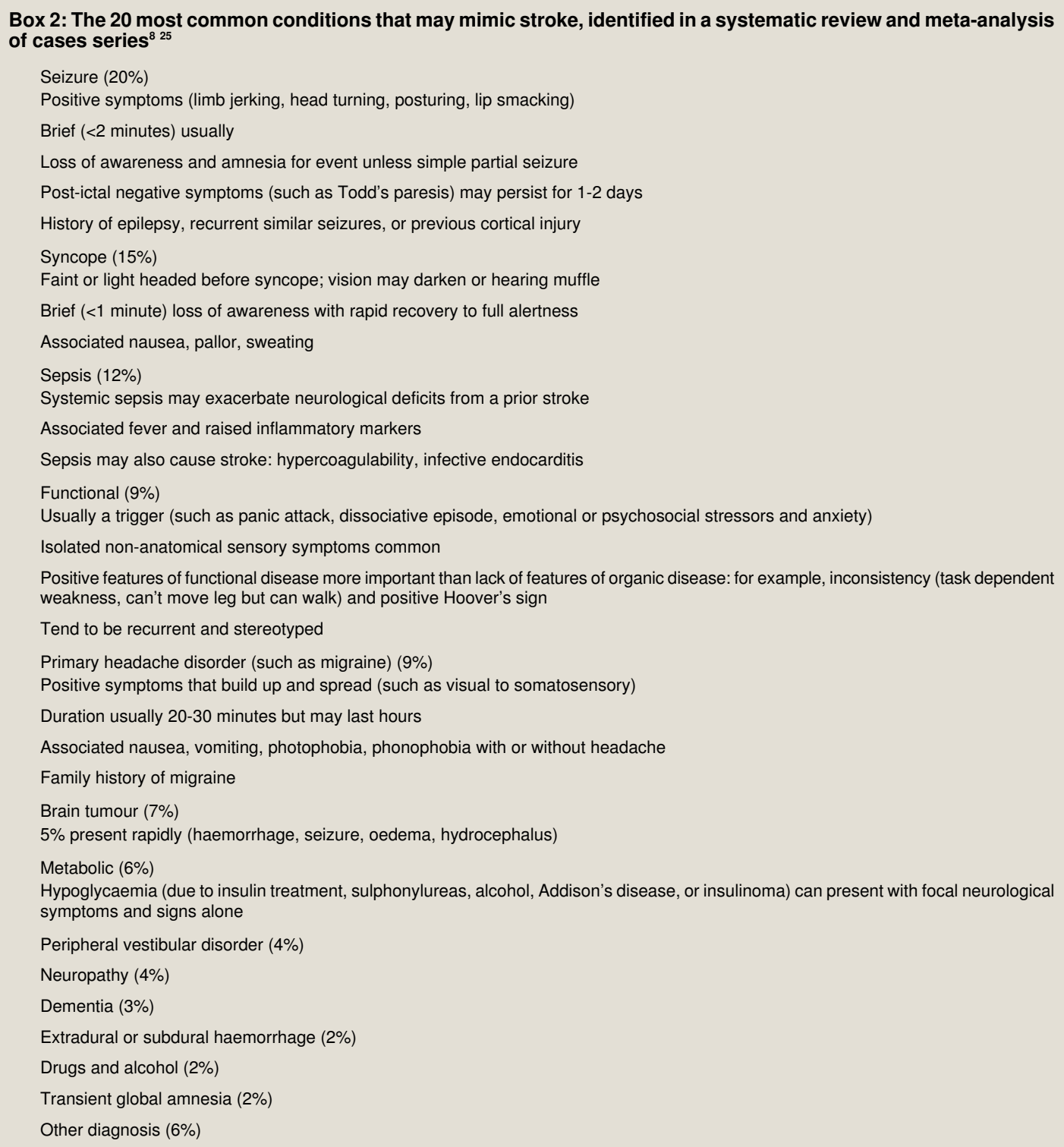

peripheral cause (such as vestibular neuritis) or a central cause (such as vestibulo-cerebellar stroke). A focused history and physical examination are often required to diagnose acute vestibular syndrome due to a vertebrobasilar stroke because the lesion is often too small to be seen on brain imaging. ${ }^{26} \mathrm{~A}$ three-step bedside oculomotor examination (HINTS: Head Impulse test, Nystagmus, Test of Skew) to elicit a normal horizontal head impulse test, direction-changing nystagmus in eccentric gaze, or skew deviation (vertical ocular misalignment) identifies stroke with high sensitivity (100\%) and specificity $(96 \%)$ in patients with acute vestibular syndrome and is more sensitive than early MRI. ${ }^{27}$

The head impulse test assesses the vestibular ocular reflex. The patient focuses on a visual target (such as the examiner's nose), and the examiner turns the patient's head to one side with a high acceleration but low amplitude head thrust. Normally, head movement towards a semicircular canal will activate that canal and evoke reflex movement of both eyes in the opposite direction, away from the canal, to maintain ocular fixation on the visual target. This reflex is preserved, and the test is negative, in patients with central causes of vertigo (such as stroke). In patients with a peripheral cause of vertigo (such as acute vestibular neuritis), head movement towards the defunct semicircular canal will fail to activate the vestibular-ocular reflex and the visual target will be lost from fixation during the sudden head movement. The patient then has to make a catch-up saccade (eye movement), usually toward the examiner's nose, to refixate the visual target, and the test is positive.

Nystagmus that changes direction on sustained gaze in one direction makes a central pathology for the acute vestibular syndrome more likely. A predominantly horizontal nystagmus that beats only in one direction and increases in intensity when the patient looks in the direction of the fast phase of the nystagmus is usually of peripheral origin.

Contributors: Both authors wrote and revised this article and approved the final version. GJH is guarantor.

Competing interests: We have read and understood the BMJ policy on declaration of interests and have no relevant interests to declare.

Provenance and peer review: Commissioned; externally peer reviewed.

1 Feigin VL, Forouzanfar MH, Krishnamurthi R, Mensah GA, Connor M, Bennett DA, et al; Global Burden of Diseases, Injuries, and Risk Factors Study 2010 (GBD 2010) and the GBD Stroke Experts Group. Global and regional burden of stroke during 1990-2010: findings from the Global Burden of Disease Study 2010. Lancet 2014;383:245-54.

2 Emberson J, Lees KR, Lyden P, Blackwell L, Albers G, Bluhmki E, et al. Effect of treatment delay, age, and stroke severity on the effects of intravenous thrombolysis with alteplase for acute ischaemic stroke: a meta-analysis of individual patient data from randomised trials. Lancet 2014;384:1929-35.

3 Hankey GJ. Secondary stroke prevention. Lancet Neurol 2014;13:178-94. 
4 Warlow C, van Gijn J, Dennis M, Wardlaw J, Bamford J, Hankey G, et al. Stroke: practical management. 3rd ed. Blackwell Publishing, 2008.

5 Sacco RL, Kasner SE, Broderick JP, Caplan LR, Connors JJ, Culebras A, et al. An updated definition of stroke for the 21 st century: a statement for healthcare professionals from the American Heart Association/American Stroke Association. Stroke 2013;44:2064-89.

6 Brazzelli M, Chappell FM, Miranda H, Shuler K, Dennis M, Sandercock PA, et al. Diffusion-weighted imaging and diagnosis of transient ischemic attack. Ann Neurol 2014;75:67-76.

7 Edlow JA, Selim MH. Atypical presentations of acute cerebrovascular syndromes. Lancet Neurol 2011;10:550-60.

8 Fernandes PM, Whiteley WN, Hart SR, Al-Shahi Salman R. Strokes: mimics and chameleons. Pract Neurol 2013;13:21-8.

9 Hankey GJ, Nelson MR. Easily missed? Subarachnoid haemorrhage. BMJ 2009;339:b2874.

10 Saposnik G, Barinagarrementeria F, Brown RD Jr, Bushnell CD, Cucchiara B, Cushman $\mathrm{M}$, et al. Diagnosis and management of cerebral venous thrombosis: a statement for healthcare professionals from the American Heart Association/American Stroke Association. Stroke 2011;42:1158-92.

11 Goldstein LB, Simel DL. Is this patient having a stroke? JAMA 2005;293:2391-402.

12 Harbison J, Hossain O, Jenkinson D, Davis J, Louw SJ, Ford GA. Diagnostic accuracy of stroke referrals from primary care, emergency room physicians, and ambulance staff using the face arm speech test. Stroke 2003;34:71-6.

13 Nor AM, McAllister C, Louw SJ, Dyker AG, Davis M, Jenkinson D, et al. Agreement between ambulance paramedic- and physician-recorded neurological signs with Face Arm Speech Test (FAST) in acute stroke patients. Stroke 2004;35:1355-9.

14 Nor AM, Davis J, Sen B, Shipsey D, Louw SJ, Dyker AG, et al. The Recognition of Stroke in the Emergency Room (ROSIER) scale: development and validation of a stroke recognition instrument. Lancet Neurol 2005;4:727-34.

15 Whiteley WN, Wardlaw JM, Dennis MS, Sandercock PA. Clinical scores for the identification of stroke and transient ischaemic attack in the emergency department: a cross-sectional study. J Neurol Neurosurg Psychiatry 2011;82:1006-10.

16 Hand PJ, Kwan J, Lindley RI, Dennis MS, Wardlaw JM. Distinguishing between stroke and mimic at the bedside: the brain attack study. Stroke 2006;37:769-75.

17 National Institute of Neurological Disorders and Stroke. NIH Stroke Scale. www.ninds. nih.gov/doctors/NIH_Stroke_Scale.pdf.
18 Bamford J, Sandercock P, Dennis M, Burn J, Warlow C. Classification and natural history of clinically identifiable subtypes of cerebral infarction. Lancet 1991:337:1521-6.

19 Merino JG, Luby M, Benson RT, Davis LA, Hsia AW, Latour LL, et al. Predictors of acute stroke mimics in 8187 patients referred to a stroke service. J Stroke Cerebrovasc Dis 2013;22:e397-403

20 Chalela JA, Kidwell CS, Nentwich LM, Luby M, Butman JA, Demchuk AM, et al. Magnetic resonance imaging and computed tomography in emergency assessment of patients with suspected acute stroke: a prospective comparison. Lancet 2007:369:293-8.

21 Brazzelli M, Sandercock PA, Chappell FM, Celani MG, Righetti E, Arestis N, et al. Magnetic resonance imaging versus computed tomography for detection of acute vascular lesions in patients presenting with stroke symptoms. Cochrane Database Syst Rev 2009;(4):CD007424.

22 Doubal FN, Dennis MS, Wardlaw JM. Characteristics of patients with minor ischaemic strokes and negative MRI: a cross-sectional study. J Neurol Neurosurg Psychiatry 2011;82:540-2.

23 Yong AW, Morris Z, Shuler K, Smith C, Wardlaw J. Acute symptomatic hypoglycaemia mimicking ischaemic stroke on imaging: a systemic review. BMC Neurol 2012;12:139.

24 Mair G, Wardlaw JM. Imaging of acute stroke prior to treatment: current practice and evolving techniques. Br J Radiol 2014;87:20140216.

25 Gibson LM, Whiteley W. The differential diagnosis of suspected stroke: a systematic review. J R Coll Physicians Edinb 2013;43:114-8.

26 Tarnutzer AA, Berkowitz AL, Robinson KA, Hsieh YH, Newman-Toker DE. Does my dizzy patient have a stroke? A systematic review of bedside diagnosis in acute vestibula syndrome. CMAJ 2011;183:E571-92.

27 Kattah JC, Talkad AV, Wang DZ, Hsieh YH, Newman-Toker DE. HINTS to diagnose stroke in the acute vestibular syndrome: three-step bedside oculomotor examination more sensitive than early MRI diffusion-weighted imaging. Stroke 2009;40:3504-10.

\section{Accepted: 24 November 2014}

Cite this as: BMJ 2015;350:h56

(c) BMJ Publishing Group Ltd 2015 


\section{Table}

Table 1 Independent predictors of the diagnosis of stroke among $\mathbf{3 5 0}$ presentations by $\mathbf{3 3 6}$ patients with suspected stroke to an urban teaching hospital in the UK, of which $241(69 \%)$ were given a final diagnosis of stroke and $109(31 \%)$ a diagnosis of stroke mimic ${ }^{16}$

Variable

Odds ratio $(95 \% \mathrm{Cl})$

Higher odds of stroke

\begin{tabular}{lc}
\hline An exact onset could be determined & $2.59(1.30$ to 5.15$)$ \\
\hline Definite history of focal neurological symptoms & $7.21(2.48$ to 20.93$)$ \\
\hline Any abnormal vascular findings ${ }^{*}$ & $2.54(1.28$ to 5.07$)$ \\
\hline NIHSSt: & 1.0 (reference) \\
\hline 0 & $1.92(0.70$ to 5.23$)$ \\
\hline $1-4$ & $3.14(1.03$ to 9.65$)$ \\
\hline $5-10$ & $7.23(2.18$ to 24.05$)$ \\
\hline$>10$ & $2.03(0.92$ to 4.46$)$ \\
\hline Signs could be lateralized to left or right side of brain & $5.09(2.42$ to 10.70$)$ \\
\hline Lower odds of stroke & 0.33 (0.14 to 0.76$)$ \\
\hline Known cognitive impairment & $0.44(0.23$ to 0.85$)$ \\
\hline Abnormal findings in any other system§ &
\end{tabular}

Abnormal findings in any other system§

*Systolic blood pressure $>150 \mathrm{~mm} \mathrm{Hg}$, atrial fibrillation, valvular heart disease, or absent peripheral pulses.

†The NIHSS (National Institute of Health Stroke Scale) is a scale of neurological impairments such as level of consciousness, ocular gaze, visual fields, speech and language function, inattention, motor and sensory impairments, and ataxia that is used to grade stroke severity (not diagnose stroke or its mimics); a higher score reflects a greater number and severity of neurological impairments. ${ }^{17}$

$\ddagger$ The OCSP (Oxfordshire Community Stroke Project) classification comprises four clinical stroke syndromes (total anterior circulation syndrome, partial anterior circulation syndrome, lacunar syndrome, posterior circulation syndrome) that are based on clinical features of the stroke. ${ }^{18}$

§Respiratory, abdominal, or other abnormal signs. 